\title{
PARAgONIMUS Y PARAgONIMIASIS EN EL NORTE PERUANO. INFECCION NATURAL DE PSEUDOTHELPHUSA CHILENSIS POR METACERCARIAS DE PARAGONIMUS BRAUN, $1899 .\left(^{*}\right)$
}

\author{
César Cuba, Nicanor lbáñez H., Eduardo Fernández y Hernán Miranda. \\ Departamento de Microbiología y Parasitología. \\ Universidad Nacional de Trujillo, Trujillo, Perú,
}

S U M A R IO

Se ha estudiado el índice de infección notural y lo intensidad del parasitismo en congrejos recolectados en tres provincias del Departamento de Cajamarco, Perú, habiéndose encontrado metacercarias de Paragonimus Braun, 1899 en el $43.97 \%$ de 539 especimenes de Pseudothelphusa chilensis Milne Edwards, 1843, único cangrejo hallado en la zona. El órgano parasitado fue casi exclusivamente el hepatopáncreos, siendo 10.33 el número promedio de metacercarias por cangrejo.

Las formas adultos logradas mediante inoculación de los metacercarias, fueron identificadas como Paragonimus peruvianus Miyozoki, lbánez y Mirando, 1969, con excepción de tres ejemplores que correspondieron a lo especie Paragonimus caliensis Little, 1968.

\section{S U M M A Y}

The index of notural infection and the density of parasitism in crabs collected in three provinces of the Departament of Cajamarco, Perú, hove been studied, and they have revealed that metacercariae of $\mathrm{Pa}$ ragonimus Broun, 1899 are found in $43.97 \%$ of 539 specimens of Pseudothelphusa chilensis, Milne Edwards, 1843, the unique crab found in the zone. The porasited organ was almost exclusively the hepatopancreas overaging 10.33 metocercarios per crab.

The adult specimens which were inoculated with metacercarioe were identified os Paragonimus peruvia. nus Miyozaki, Miranda and lbonez, 1969, with the exception of three somples wich belonging to the species Paragonimus caliensis Little, 1968.

\section{INTRODUCCION}

En diversas áreas de América en las que el parasitismo por trematodos pertenecientes al género Paragonimus ha sido demostrado en el hombre y los animales, se han realizado estudios tendientes a identificar a los hospedadores intermediarios del parásito; es asi que existen algunas informaciones sobre crustáceos encontrados naturalmente infectados. $\mathrm{Fi}$ guran entre ellos, Pseudothelphusa tristani (Sogandares-Bernal y Samalley, 1965); Pseudothelphusa (Ptycophallus) tristani y Pseudothelphusa (Megathelphusa) magna (Brenes y col., 1968) en Costa Rica. En Colombia fue señalada Strengeria (Strengeria) sp. (Little, 1968).

En el Perú fue demostrada la presencia de metacercarias correspondientes a Paragonimus peruvianus en cangrejos de la especie Pseudothelphusa chilensis (Grados y cols., 1968; Grados y cols.,
1972; Ibáñez, 1970) crustáceos muy abundantes en las zonas endémicas descritas en el norte peruano. (Grados y cols., 1968; Miranda y cols., 1967; Ibáñez, 1970). Sin embargo, las observaciones referentes al grado de intensidad del parasitismo son fragmentarias e incompletas, razón por la cual decidimos realizar un estudio cuyos objetivos fueron:

a) Determinar el grado de parasitismo de los crustáceos en zonas conocidas como endémicas y en otras de caracteres ecológicos similares a las de aquéllas.

b) Precisar el número y localización de las metacercarias de Paragonimus en los diversos órganos internos del crustáceo anotando el sexo y tamaño del hospedador, además de la zona de procedencia del mismo.

c) Disponer de metacercarias para fines experimentales destinados a obte-

(*) El presente estudio ha sido realizado con el apoyo de la Universidad Nacional de Trujillo y mediante un fondo otorgodo por el Instituto Hipólito Unanue de Lima, Perú. 
ner y estudiar las formas adultas correspondientes.

\section{MATERIAL Y METODOS}

El órea geogrófica en la que se desarrolló el trabajo abarca localidades del Departamento de Cajamarca situadas en las vertientes de las cordilleras Occidental y Central de los Andes peruanos. (Fig. 7).

La altura máxima correspondiente a "San Juan" que se encuentra a 2,200 m. s.n.m. y la más baja a "Nanchoc" que estó a 500 m.s.n.m.

La mayor parte de las áreas visitadas son rurales y unas pocas son caseríos de pequeña población.

Los habitantes se dedican generalmente a la pequeña agricultura y basan su alimentación en recursos naturales que incluyen los cangrejos de agua dulce que ingieren a menudo, crudos o mal cocidos.

Las localidades corresponden a las provincias de Cajabamba, Cajamarca y San Miguel. En la primera fueron estudiadas "La Granja" o "Chaquicocha", Los Naranjos, Pomabamba, Santa Elena, Nuñumabamba, que estón en la margen derecha del río Condebamba (Fig. 1); Chuquibamba, Santa Cristina, Chingol, Tabacal, Chimín (que corresponde a "Cueva del Oso") ubicados en la margen izquierda.

En la provincia de Cajamarca se trabajó en San Juan, San Marcos (Saparcón) y La Asunción.

En la provincia de San Miguel se investigó en Bolívar, Nanchoc, La Tambora, El Sauce, El Diamante, localidades que pertenecen al distrito de Nanchoc.

Los cangrejos han sido frecuentemente encontrados en acequias de regadio de caña de azúcar, maiz, yuca y otras plantaciones, en las riberas y "playas" de los afluentes de los ríos interandinos y debajo de las piedras en las quebradas (Fig. 2).

El habitat consiste en trayectos de profundidad variable que el animal excava en la tierra húmeda, los que la gente identifica con el nombre de "cangrejeras".

Los especímenes fueron capturados vivos y transportados al laboratorio; se les paralizaba mediante la destrucción de la cadena nerviosa ganglionar ventral con un estilete y hecha la medición del cefalotórax y la determinación del sexo, se levantaba la cubierta exponiendo los órganos internos.

La investigación de las metacercarias se practicó en cada ejemplar mediante estereomicroscopio, presionando los órganos entre láminas de vidrio y observando por separado el hepatopáncreas, corazón, genitales, branquias y músculos de las quelas, anotando el número de metacercarias por órgano y el total de formas larvarias obtenidas por cangrejo, tratando de evitar transferir metacercarias de un órgano a otro en el proceso de la disección.

Se trabajó también en especímenes que resultaron muertos durante el transporte, no habiéndose hecho una separación estricta de este grupo con relación a los crustáceos vivos.

\section{RESULTADOS}

El número de cangrejos examinados en total, ascendió a 539 distribuidos en la siguiente forma:

a) Provincia de Cajabamba: 302 .

b) Provincia de Cajamarca: 109.

c) Provincia de San Miguel: 128.

De los 539 cangrejos, estuvieron parasitados por metacercarias de Paragonimus $237(43.97 \%)$ obteniéndose en ellos un total de 2,450 metacercarias.

El promedio de metacercarias por cangrejo, considerado el grupo parasita. do, fue de 10.33. Si se considera el total de cangrejos, dicho promedio desciende a 4.50 por cangrejo.

Al analizar la distribución del número de metacercarias considerando su frecuencia, observamos que el $58.64 \%$, es decir, 139 de 237 cangrejos tenían de 1 a 5 metacercarias por ejemplar.

$42(17.72 \%)$ tenían de 6 a 10 formas larvarias. $15(6.32 \%)$ presentaron de 11 
a 15 y $12(5.06 \%)$ entre 16 a 20 por cangrejo. Menor número de cangrejos fueron observados con cifras mayores de metacercarias; habiéndose llegado en un caso hasta 161 , en otro hasta 118 , en un tercero a 91, en otros a $83,78,73,61$ y 51 , en cada uno. (Tabla 2).

En el cangrejo que exhibió el número máximo, las larvas estaban ubicadas en su totalidad en el hepatopáncreas, el especimen era hembra y medía $6 \mathrm{~cm}$. de ancho en el cefalotórax y procedía de la localidad denominada "Cueva del Oso" en Chimín. Cabe anotar que los crustáceos observados alcanzan dimensiones que oscilan entre 2 y $9 \mathrm{~cm}$. de ancho.

El hepatopáncreas fue el órgano parasitado por excelencia. En forma excepcional se observaron metacercarias en el corazón ( 5 casos), en ovarios ( 3 casos), en músculos de las quelas (5 casos) $y$ en branquias ( 1 caso).

La totalidad de los crustáceos estudiados pertenecen a la especie Pseudothelphusa chilensis Milne Edwards, 1843 (Del Solar y cols., 1970) (Fig. 3), de ellas, 270 fueron hembras y 259 machos.

El mayor número de cangrejos parasitados eran hembras. La mayor parte de los datos expuestos aparecen en la Tabla 1. Las metacercarias encontradas carecían de envoltura quística y presentaban, hasta donde fue posible observar, las características de Paragonimus peruvianus (Miyazaki, 1972). Figs. 4 y 5.

Se ha observado buena movilidad de las metacercarias en cangrejos muertos aún en aquéllos en estado de putrefacción.

Las formas adultas resultantes de inocular las metacercarias en gato doméstico, fueron identificadas como Paragonimus peruvianus Miyazaki, lbáñez y Miranda, 1969, con excepción de 3 especímenes que correspondieron a la especie P. caliensis Little, 1968.

\section{DISCUSION}

Los conocimientos sobre Paragonimus y Paragonimiasis en el Perú, han sido considerablemente incrementados en años recientes. Sin embargo, nos pareció necesario ahondar aquellos aspectos relati- vos al segundo huésped intermediario mediante estudios respaldados por la observación de un número significativo de especímenes colectados en diversas áreas.

Cumplidas las acciones programadas se ha determinado que la única especie de crustáceo encontrada por nosotros, naturalmente parasitada por metacercarias de Paragonimus, es Pseudothelphusa chilensis, Milne Edwards, 1843, en las zonas consignadas en este trabajo.

Es particularmente llamativo el alto porcentaje de infección natural que presenta la especie nombrada $(43.97 \%$ ) y lo es igualmente, el alto número de formas larvarias que en promedio se encuentran en cada carigrejo (10.33). Del análisis de la Tabla 2 se desprende que las metacercarias por cangrejo alcanzan cifras que consideramos muy elevadas, al comparar éstas con las obtenidas por otros autores en otros crustáceos (Brenes y cols., 1968; Little, 1968); lo que explicaria la importancia de P. chilensis en la cadena epidemiológica de la paragonimiasis en las áreas estudiadas.

Ctro aspecto de importancia se refiere a la localización de las larvas casi exclusivamente en el hepatopóncreas. Creemos que su hallazgo en localizaciones distintas se deba a migraciones, no pudiéndose descartar posibles defectos en el aislamiento de los órganos internos durante la disección.

Observaciones realizadas por otros autores en otros países de América, nos permiten apreciar mejor el significado de los resultados registrados.

Sogandares-Bernal y Smalley (1965) en Costa Rica reportaron el hallazgo de una metacercaria de Paragonimus sp. en un ejemplar de Pseudothelphusa tristani. Thatcher (1967) en Panamá encontró 3 matacercarias de Paragenimus en las branquias de Pseudothelphusa richmondi. Brenes, Zeledón y Rojas (1968) examinaron 150 cangrejos de la especie Pseudoshelphusa (Prycophallus) tristani colectados en Costa Rica y encontraron 19 especímenes parasitados por metacercarias de Paragonimus sp. (12.7\%). De 5 ejemplares de Pseudothelphusa (Megathelphusa) magna uno fue encontrado parasitado. 
Little (1968) investigando en áreas en las que se encontraron mamiferos silvestres parasitados con Paragonimus caliensis, demostró que el crustáceo Strengeria (Strengeria) sp. estaba parasitado por metacercarias de la especie nombra. da en un $5.1 \%$, habiendo examinado 520 especimenes.

El mayor número de metacercarias por cangrejo encontradas por este autor fue de 7, siendo el promedio 2.4 metacercarias por cangrejo.

Yokogawa (1971) encontró una metacercaria de Paragonimus en uno de los 18 cangrejos de la especie Strengeria engeymani colectados en las localidades de Rob!es y Caluma en el Ecuador. El ejemplar parasitado procedía de Caluma.

Al comparar los resultados nuestros con los de los autores mencionados, vemos que el porcentaje de parasitismo y el número de metacercarias por cangrejo, alcanzan cifras muy elevadas en comparación con las registradas por los autores nombrados. Siendos estos crustáceos comúnmente usados como alimento por los pobladores de las zonas estudiadas, es incuestionable su gran significado epidemiológico. Por lo expuesto, no es de extrañar que la Paragonimiasis humana resulte ser una enfermedad frecuente entre dichos pobladores, tal como ha sido comprobado en otros trabajos (Miranda y cols., 1967; Cuba, 1971; Grados y cols., 1972); y confirmado por nuestras observaciones. Esta situación se explica por el hecho de que los cangrejos son ingeridos insuficientemente cocidos o sin cocción alguna.

Se ha registrado igualmente que los pobladores del lugar atribuyen carácter medicinal a bebidas y otras preparaciones obtenidas de cangrejos, todo lo cual eleva las posibilidades de infección humana.

Un aspecto que asume, a nuestro juicio, una significación especial se refiere a la localidad denominada "Cueva del Oso" en el área de Chimín en la que se capturaron 9 cangrejos, todos los cuales resultaron intensamente parasitados, portando uno de ellos 161 metacercarias.

La localidad en referencia se encuentra en la parte más alta de un cañón an- gosto por el que desciende una acequia que en la parte investigada se encuentra a cerca de 2 kilómetros de distancia de las zonas habitadas más próximas y no es transitable en razón de estar en una pendiente muy marcada de difícil acceso. El hecho de que la presencia humana sea tan infrecuente en esa zona, aparejado a un alto índice de parasitismo en los cangrejos nos permite deducir que el ciclo del parásito puede ser mantenido por lo menos en ciertos lugares, por los carnivoros silvestres, sin intervención humana.

Si bien no es finalidad esencial del presente trabajo referimos a las formas de Paragonimus, es pertinente hacer un breve comentario al respecto. Según lbáñez y cols. (1974) las formas adultas extrádas de pulmones de gatos inoculados con meiacercarias, corresponden a la especie aragaimus peruvianus casi en su totalidad. Una proporción mínima, representada por 3 ejemplares, resultaron ser P. caliensis. Se infiere de ello que Pseudothelphusa chilensis es también huésped intermediario de P. caliensis en el Perú; las metacercarias de esta especie procedieron de las localidades de Sunchubamba y Chingol, de la Provincia de Cajabamba. Estos hechos no han sido registrados con anterioridad, hasta donde estamos informados. Por otro lado, es indiscutible que las metacercarias correspondientes a $\mathbf{P}$. caliensis pasaron desapercibidas en el momento de su aislamiento a partir del cangrejo, lo cual es explicable, entre otras razones, por el aran volumen de formas larvarias examinadas.

\section{REFERENCIAS BIBLIOGRAFICAS}

BRENES, R. R, ZELEDON, R. Y ROJAS G. - 1968. The finding of Paragonimus $\mathrm{sp}$. in mommals, crabs and snoils in Costa Rico. Bol. Chileno Parost. 23:164.

CUBA, C. - 1971. Paragonimiasis en el Perú: zonas endémicas de paragonimiasis determinodas hasto la actualidad. Libro de Confe rencias y Mesas Redandas del 111 Congreso Peruano de Microbiología y Parasitología, Trujillo, Perú. Pógs. 149-154.

DEL SOLAR, C. E. M., BLANCAS, S. F. Y MAYTA, L. R. - 1970. Católogo de crustáceos del Perú. Lima, Perú. 
GRADOS, O., CUBA, C. y MAZABEL, C. - 1967. Paragonimiasis en el Perú: Determinación de algunas áreas endémicas. Libro de Resúmenes del IV Congreso Latinoamericano de Microbiología y II Congreso Peruano de $\mathrm{Mi}$ crobiolgía y Parasitología. Lima, Perú. Pág. 208.

GRADOS, O., CUBA, C., MORALES, N. y MAZABEL, C. - 1972. Epidemiología de la Paragonimiasis en el Perú. Arch. Peruanos Pat. Clin. 25: 33-54.

IBAÑEZ, N. - 1970. Paragonimus peruvianus sp. n. y sus huéspedes intermediarios. Libro de Conferencias y Mesas Redondas del III Congreso Peruano de Microbiología y Parasitología. Trujillo, Perú. Págs. 155-158.

IBAÑEZ, H. N., MIRANDA, H., FERNANDEZ, E., y CUBA, C. - 1974. Paragonimus y Paragonimiosis en el Norte Peruano. II. Proceso del desarrollo de Paragonimus peruvianus Miyazaki, Ibáñez y Miranda, 1969. (Trematoda; Troglotrematidae) en Felis cati $L$. infectado experimentalmente. Rev. Per. Biol. $1: 31-56$
LITTLE, M. D. - 1968. Paragonimus caliensis SP. $\mathrm{n}$. and Paragonimiasis in Colombia. J. Parasit. 54:738-746.

MIRANDA, H., FERNANDEZ, O., MONTENEGRO, H. y ALVA, F. - 1967. Paragonimiasis. Nota sobre nuevas áreas de procedencia de portadores de la enfermedad. Arch. Peruanos Pat. Clin. 21:215-222.

MIYAZAKI, I. - 1972. Lung flukes in the Western Hemisphere. Overseas Tech. Co. Agency Tokyo. Japan.

SOGANDARES-BERNAL, $F$. and SMALLEY, A. E. - 1965. Paragonimus metacercarie in Pseudothelphusa tristani Rathbun from Costa Rica. J. Parasit. $51: 304$.

THATCHER, V. E. - 1967. Paragonimus in some wild and domestic animals of Panamá. Trans. Amer. Microsc. Soc. 86:335-336.

YOKOGAWA, M. - 1971. Report on a field visit to Ecuador and Perú. 1-15. Texto mimeografiado. 


\section{TABLA 1}

INFECCION NATURAL DE 539 ESPECIMENES DE Pseudothelphusa chilensis RECOLECTADOS EN TRES PROVINCIAS DEL DPTO. DE CAJAMARCA, PERU; TOMANDO EN CUENTA PORCENTAJE, ORGANOS PARASITADOS $Y$ PROMEDIO DE METACERCARIAS POR CANGREJO.

\begin{tabular}{|c|c|c|c|c|c|}
\hline $\begin{array}{l}\text { Lugares de } \\
\text { Procedencia }\end{array}$ & $\begin{array}{c}\text { Total de } \\
\text { Cangrejog } \\
\text { Recolectados }\end{array}$ & $\left|\begin{array}{c}\text { Wo Y Sexo de } \\
\text { Positivos } \\
\text { (macho-hembra) - }\end{array}\right|$ & $\begin{array}{c}\text { Porcentaje } \\
\text { de } \\
\text { Positivos }\end{array}$ & $\begin{array}{l}\text { No de Me- } \\
\text { tacercarias } \\
\text { por Cangr } \\
\text { (Promedio) }\end{array}$ & $\begin{array}{c}\text { Organos Parasitados } \\
\text { No do Cangrejos }\end{array}$ \\
\hline \multicolumn{6}{|l|}{$\begin{array}{l}\text { Provincia de } \\
\text { Cajabambo }\end{array}$} \\
\hline La Granja & 43 & $30(9-21)$ & 69.76 & 11.53 & $30 \mathrm{Hp} .(2 \mathrm{C} ; 1$ Or; $1 \mathrm{~B} ; 1 \mathrm{Q})$ \\
\hline Tabacal. & 109 & $79(31-48)$ & 72.47 & 11.29 & $79 \mathrm{Hp} .(2 \mathrm{C} ; 2$ Ov. $3 \mathrm{Q})$ \\
\hline Chimin & 16 & $15(3-12)$ & 93.75 & 36.73 & $15 \mathrm{Hp}$. \\
\hline Nuñumabambo & 7 & $2(2-0)$ & 28.57 & 6.50 & $2 \mathrm{Hp}$. \\
\hline Los Naranjos & 30 & $12(3-9)$ & 40.00 & 9.58 & $12 \mathrm{Hp}$. \\
\hline Pomabambo. & 7 & $0(-\longrightarrow)$ & 0.00 & 0.00 & - \\
\hline Sta. Elena & 17 & $2(2-0)$ & 11.76 & 2.00 & $2 \mathrm{Hp}$. \\
\hline Chuquibambo & 16 & $10(7-3)$ & 62.50 & 19.40 & $10 \mathrm{Hp}$. \\
\hline Chingol . . & 7 & $4(2.2)$ & 57.14 & 6.50 & $4 \mathrm{Hp}$. \\
\hline Sta. Cristina & 14 & $9(4-5)$ & 64.28 & 2.00 & $9 \mathrm{Hp}$. \\
\hline Sunchubamba & 36 & $11(9-2)$ & 30.55 & 6.27 & $11 \mathrm{Hp}$. \\
\hline \multicolumn{6}{|l|}{$\begin{array}{l}\text { Provincia de } \\
\text { Cojomarco }\end{array}$} \\
\hline San Juan. . & 86 & $7(3-4)$ & 8.13 & 12.42 & $7 \mathrm{Hp}$. \\
\hline San Marcos. & 11 & $2(1-1)$ & 18.18 & 3,00 & $2 \mathrm{Hp}$. \\
\hline Lo Asunción . . . & 12 & $0(-\rightarrow)$ & 0.00 & 0.00 & - \\
\hline \multicolumn{6}{|l|}{$\begin{array}{l}\text { Provincia de } \\
\text { San Miguel }\end{array}$} \\
\hline Bolivar. & 30 & $9(6.3)$ & 30.00 & 3.11 & $9 \mathrm{Hp.} \mathrm{(1} \mathrm{C)}$ \\
\hline Nanchoc & 51 & $18(8-10)$ & 35.29 & 2.30 & $18 \mathrm{Hp} .(1 \mathrm{Q})$ \\
\hline La Tambora & 19 & $15(5-10)$ & 78.94 & 2.73 & $15 \mathrm{Hp}$. \\
\hline EI Sauce & 16 & $7(4-3)$ & 43.75 & 1.42 & $7 \mathrm{Hp}$. \\
\hline El Diamante .... & 12 & $5(2-3)$ & 41.61 & 1.40 & $5 \mathrm{Hp}$. \\
\hline TOTALES & 539 & $237(101-136)$ & 43.97 & 10.33 & 237 Hp. (5 C; 3 Or.; 1 B; 5 Q) \\
\hline
\end{tabular}

Hp. Hepatopáncreas

c. Corazón

Q. quelas 
TABLA 2

DISTRIBUCION DEL NUMERO DE METACERCARIAS DE Paragonimus peruvianus EN 237 EJEMPLARES DE Pseudothelphusa chilensis

\begin{tabular}{c|c|c}
\hline No de Metacercarios & $\begin{array}{c}\text { Parasitados } \\
\text { No de Cangrejos }\end{array}$ & Porcentaje \\
\hline $1-5$ & 139 & 58.64 \\
$6-10$ & 42 & 17.72 \\
$11-15$ & 15 & 6.32 \\
$16-20$ & 12 & 5.06 \\
$21-25$ & 8 & 3.75 \\
$26-30$ & 7 & 3.33 \\
$31-35$ & 1 & 0.45 \\
$36-40$ & 1 & 0.45 \\
$40-45$ & 0 & 0.00 \\
$46-50$ & 4 & 1.64 \\
$51-a$ mós * & 8 & 3.75 \\
\hline
\end{tabular}

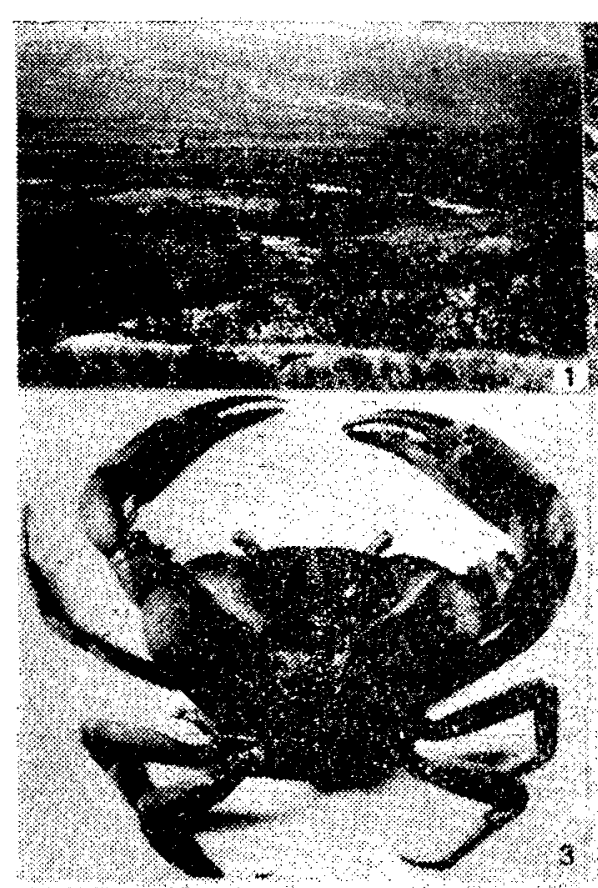

Fig. 1.-Valle de Condebamba

Fig. 2.- Hobitat coracterístico de Pseudothelphuse chilensis.

Fig. 3.-Pseudothelphusa chilensis.

Fotografías: Hernán Miranda $C$.
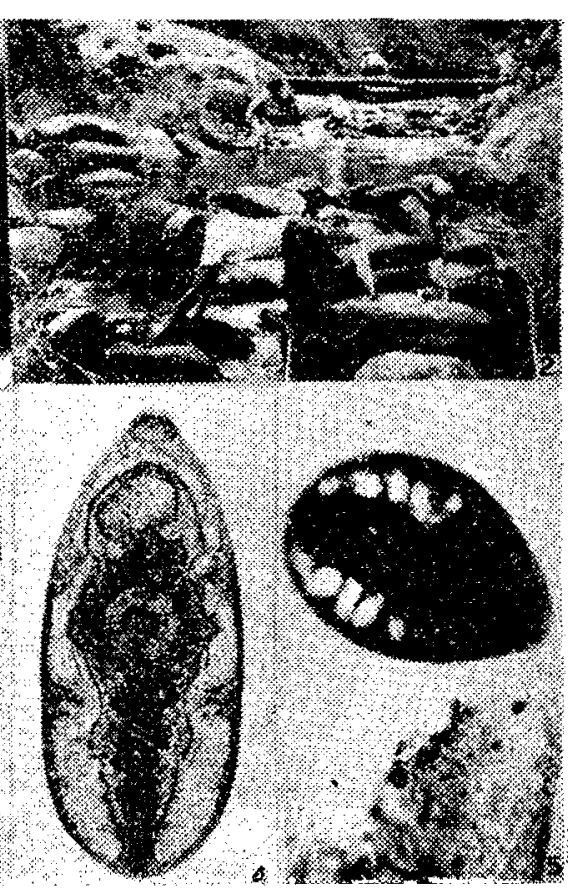

Fig. 4.-Metacercaria coloreada.

Fotografía: Wilton Soldaña S

Fig. 5.- Metacercaria recién extraida Fotogratía: Jesús Garcia A. 


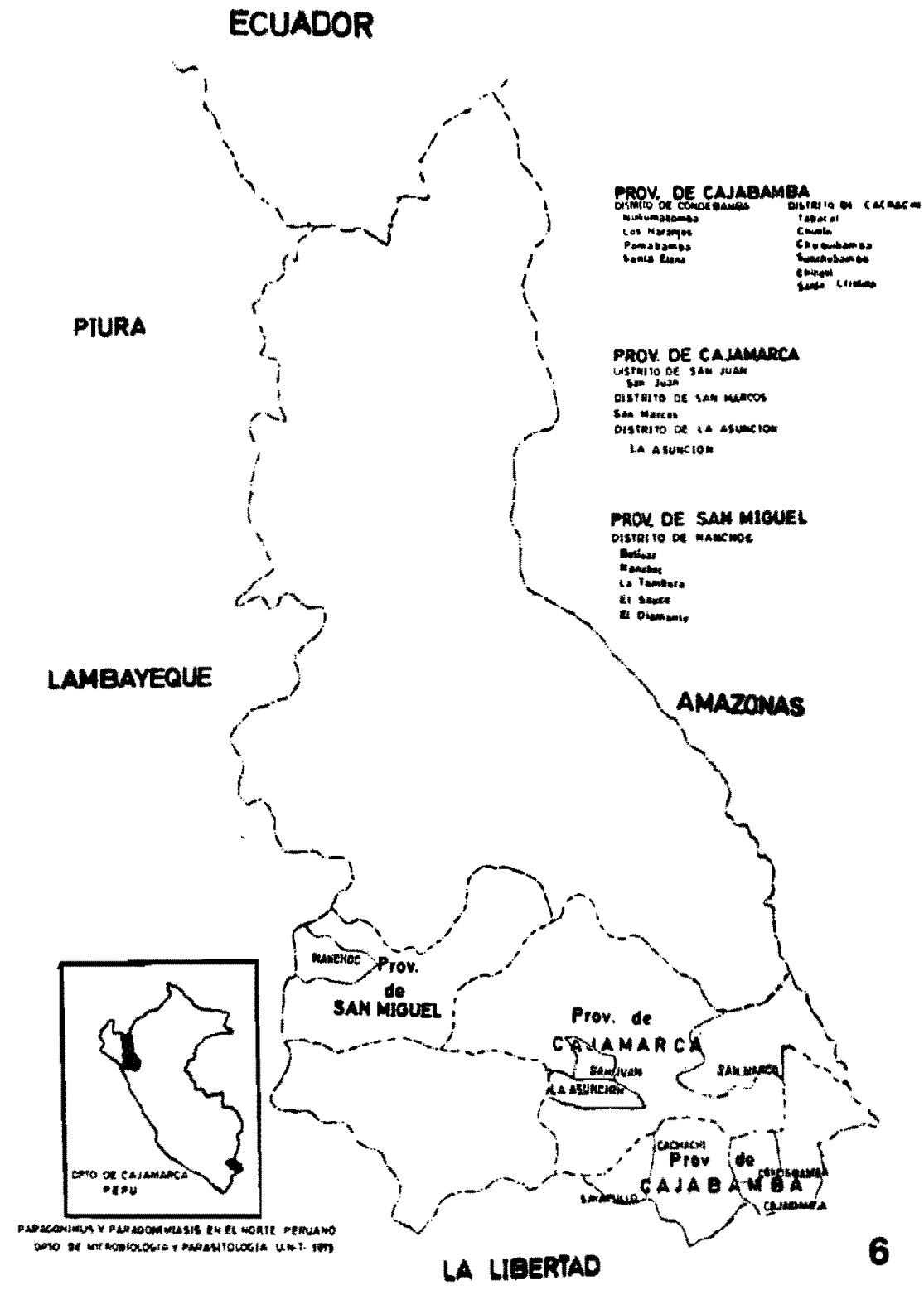

Fig. 6.-Areas estudiadas en el Dpto. de Cajomarca. 